\title{
A novel PBP3 substitution in Haemophilus influenzae confers reduced aminopenicillin susceptibility
}

\author{
John Thegerström ${ }^{1 *} \mathbb{D}$, Erika Matuschek ${ }^{2}$, Yu-Ching Su' ${ }^{1}$ Kristian Riesbeck $^{1}$ and Fredrik Resman ${ }^{1}$
}

\begin{abstract}
Background: Identification and characterization of non-typeable Haemophilus influenzae (NTHi) with reduced susceptibility to $\beta$-lactam antibiotics due to mutations in penicillin binding protein 3 (PBP3) is a clinical challenge. We analyzed a blood isolate, NTHi93-57485, that was categorized as aminopenicillin resistant but lacked key amino acid substitutions in PBP3 that have previously been associated with reduced aminopenicillin susceptibility. The significance of an alternative amino acid substitution (Y528H) in this isolate was examined.

Results: Site-directed mutagenesis of a $\beta$-lactam susceptible H. influenzae (NTHi3655) was performed to introduce the Y528H substitution into wild-type ftsl (encoding for PBP3). Disc diffusion screening and broth microdilution determination of MICS for $\beta$-lactam agents were done with the NTHi3655-PBP3 ${ }^{\text {Y528H }}$ mutant and were compared with the NTHi3655 wild-type as well as the original clinical isolate NTHi93-57485. Introduction of the Y528H substitution in NTHi3655 resulted in positive screening for $\beta$-lactam resistance. MICs for aminopenicillins were increased in the mutant compared to the wild-type. However, the mutant remained susceptible to aminopenicillins according to EUCAST clinical breakpoints (assuming intravenous treatment) and the introduction of $\mathrm{Y} 528 \mathrm{H}$ alone did not increase the resistance to the same level as NTHi93-57485. None of the isolates had frame shift insertions in the acrR gene regulating the AcrAB efflux pump.
\end{abstract}

Conclusions: In parallel to the previously well-described PBP3-substitutions R517H and N526K, we demonstrate that Y528H confers reduced aminopenicillin susceptibility.

Keywords: Ampicillin - $\beta$-lactam resistance - ftsl - Haemophilus influenzae - PBP3 - penicillin binding proteins - site directed mutagenesis

\section{Introduction}

Antimicrobial resistance of the respiratory tract pathogen non-typeable Haemophilus influenzae (NTHi) to $\beta$-lactam antibiotics is conferred either by the production of transferrable $\beta$-lactamases or by amino acid substitutions in penicillin binding protein 3 (rPBP3), caused by point mutations of the ftsI gene [1]. It has also been shown that loss of repression of the AcrAB efflux pump in combination with rPBP3 may lead to a further increase in resistance [2].

NTHi strains with rPBP3 variants are classified into three main groups (Table 1), based on the substitution of two key amino acids occurring near the KTG-motif: R517H (clustered as group I) or N526K (group II) [3]. A

\footnotetext{
*Correspondence: john.thegerstrom@med.lu.se

${ }^{1}$ Clinical Microbiology, Department of Translational Medicine, Lund

University, Jan Waldenströms gata 59, SE-205 02 Malmö, Sweden

Full list of author information is available at the end of the article
}

third group with additional substitutions near the SSN-motif, S385T (group III or III-like) confers a higher-level of antimicrobial resistance, including resistance to third-generation cephalosporins [3-5]. The group II-rPBP3 variants can be further categorized into the subgroups IIa-d or A-G, depending on the pattern of mutations within ftsI that appear together with N526K $[6,7]$. The evidence of correlation between these key substitutions and resistance phenotype is strong [3, 8], but the causal evidence of these substitutions as single determinants of resistance is less convincing. When Osaki and co-authors applied site-directed mutagenesis to introduce PBP3 substitutions into a $\beta$-lactam susceptible strain ( $H$. influenzae $\mathrm{Rd}$ ), neither the introduction of $\mathrm{R} 517 \mathrm{H}$ nor N526K could alone generate mutants that were aminopenicillin resistant (ampicillin $\mathrm{MIC}=0.25 \mathrm{mg} / \mathrm{L}$ for $\mathrm{N} 526 \mathrm{~K}$ ) according to

(c) The Author(s). 2018 Open Access This article is distributed under the terms of the Creative Commons Attribution 4.0 International License (http://creativecommons.org/licenses/by/4.0/), which permits unrestricted use, distribution, and 
Table 1 The principal groups of rPBP3 in Haemophilus influenzae with their associated amino acid substitutions and susceptibility to ampicillin. The clinical breakpoint for ampicillin is definied as $R>1 \mathrm{mg} / L$ by EUCAST, which means that a subset of NTHi with rPBP3 genotype are still categorized as susceptible. Table modified after Skaare et al. [7]

\begin{tabular}{|c|c|c|c|c|c|}
\hline $\begin{array}{l}\text { Main rPBP3 } \\
\text { group }\end{array}$ & $\begin{array}{l}\text { Subgroup according } \\
\text { to Skaare [7] }\end{array}$ & $\begin{array}{l}\text { Subgroup according to } \\
\text { Ubukata [3] and Dabernat [6] }\end{array}$ & $\begin{array}{l}\text { Key amino acid } \\
\text { substitutions }\end{array}$ & $\begin{array}{l}\text { Associated substitutions } \\
\text { in subgroups }\end{array}$ & $\begin{array}{l}\text { MIC range of } \\
\text { Ampicillin }(\mathrm{mg} / \mathrm{L})\end{array}$ \\
\hline Group I & & & $\mathrm{R} 517 \mathrm{H}$ & & $0.5-2[5]$ \\
\hline \multirow[t]{8}{*}{ Group ॥ } & & & N526K & & $0.5-8[5]$ \\
\hline & A & $\| \mathrm{b}$ & N526K & D350N M377I A502V V547I N569S & \\
\hline & B & $\| d$ & N526K & I449V V547I N569S & \\
\hline & C & $\| \mathrm{b}$ & N526K & D350N M377I G490E A502V V547I N569S & \\
\hline & $\mathrm{D}$ & $\|-$ & N526K & D350N G490E A530S & \\
\hline & $E$ & IIc & N526K & A502T & \\
\hline & $\mathrm{F}$ & $\| a$ & N526K & - & \\
\hline & G & $\|-$ & N526K & V547I A554T A561E N569S & \\
\hline Group III & & & $\mathrm{S} 385 \mathrm{~T}+\mathrm{N} 526 \mathrm{~K}$ & & $1-32[4]$ \\
\hline Group III-like & & & $\mathrm{S} 385 \mathrm{~T}+\mathrm{R} 517 \mathrm{H}$ & & $0.5-2[5]$ \\
\hline
\end{tabular}

clinical breakpoints, although a reduced susceptibility compared to the wild-type (WT) isolate was seen [9].

A screening algorithm to identify rPBP3 strains in routine diagnostics based on disc diffusion with $1 \mathrm{U}$ benzylpenicillin is suggested by the European Committee on Antimicrobial Susceptibility Testing (EUCAST) [10]. This screening algorithm has demonstrated high sensitivity and specificity in detecting rPBP3 isolates, although confirmatory testing of actual MIC levels is recommended to determine if screening-positive isolates are actually resistant [8, 11]. Since current clinical breakpoints for aminopenicillins split the rPBP3 group, this implies that a subset of rPBP3 isolates are still considered susceptible [8] (Table 1). Moreover, the breakpoints assume intravenous dosage, and therefore there is debate on the optimal treatment of these strains in infections that do not require intravenous antibiotic therapy.

In the present study, we investigated a clinical NTHi isolate that was aminopenicillin resistant according to initial disc diffusion screening and MIC determination, but lacked resistance-defining substitutions in PBP3, and instead had an alternative substitution near the KTG-motif; Y528H.

\section{Methods}

\section{Bacterial strains and culture conditions}

A clinical blood isolate from Kronoberg County (Sweden) (NTHi93-57485) was collected as part of routine diagnostics at the laboratory of clinical microbiology in Växjö, Sweden. The isolate was screened as $\beta$-lactam resistant by disc diffusion, was $\beta$-lactamase negative by nitrocefin testing and had an MIC for amoxicillin of $2 \mathrm{mg} / \mathrm{L}$ according to the initial Etest (bioMérieux, Marcy l'Etoile, France), and thus aminopenicillin resistant according to EUCAST clinical breakpoints at the time
(2011). The isolate did not, however, carry any of the previously described key mutations in ftsI and was therefore chosen for further testing. DNA sequencing of $f_{s t} I$ revealed an alternative amino acid substitution located near the KTG-motif; Y528H (Fig. 1).

For site-directed mutagenesis, a well-characterized, $\beta$-lactam-susceptible isolate (NTHi3655) previously kindly donated by R. Munson, St Louis, Mo., was chosen [12]. This strain was chosen since its full genome sequence is known and it has a wtPBP3 sequence identical to that of H.influenzae Rd. (accession no. AAZF01000004.1). ATCC49766 (American Type Culture Collection, LGC standards, Teddington, UK) was used as quality control in the antimicrobial susceptibility testing. All strains were cultured on chocolate agar or in brain heart infusion (BHI) broth supplemented with $10 \mu \mathrm{g} / \mathrm{ml}$ each of nicotinamide adenine dinucleotide (NAD) and hemin overnight at $37^{\circ} \mathrm{C}$ and $5 \% \mathrm{CO}_{2}$. All isolates were confirmed as Haemophilus influenzae by Matrix Assisted Laser Desorption Ionization Time of Flight (MALDI-TOF, scores $>2$ ).

Only microorganisms and no human material were handled in this project.

\section{Site-directed mutagenesis (SDM)}

Genomic DNA was purified using the GenElute ${ }^{\mathrm{rm}}$ Bacterial Genomic DNA kit (Sigma-Aldrich, St Louis, MO). The ftsI gene and its flanking regions were amplified from the WT NTHi3655 by using the Expand ${ }^{\mathrm{TM}}$ High Fidelity PCR System (Roche, Mannheim, Germany) and primers listed in Table 2.

The resulting PCR product was cloned into the pCR-XL-TOPO vector by using TOPO $^{\circ} \mathrm{XL}$ PCR cloning kit (Invitrogen, Carlsbad, CA). The recombinant plasmid construct was thereafter transformed into Escherichia 


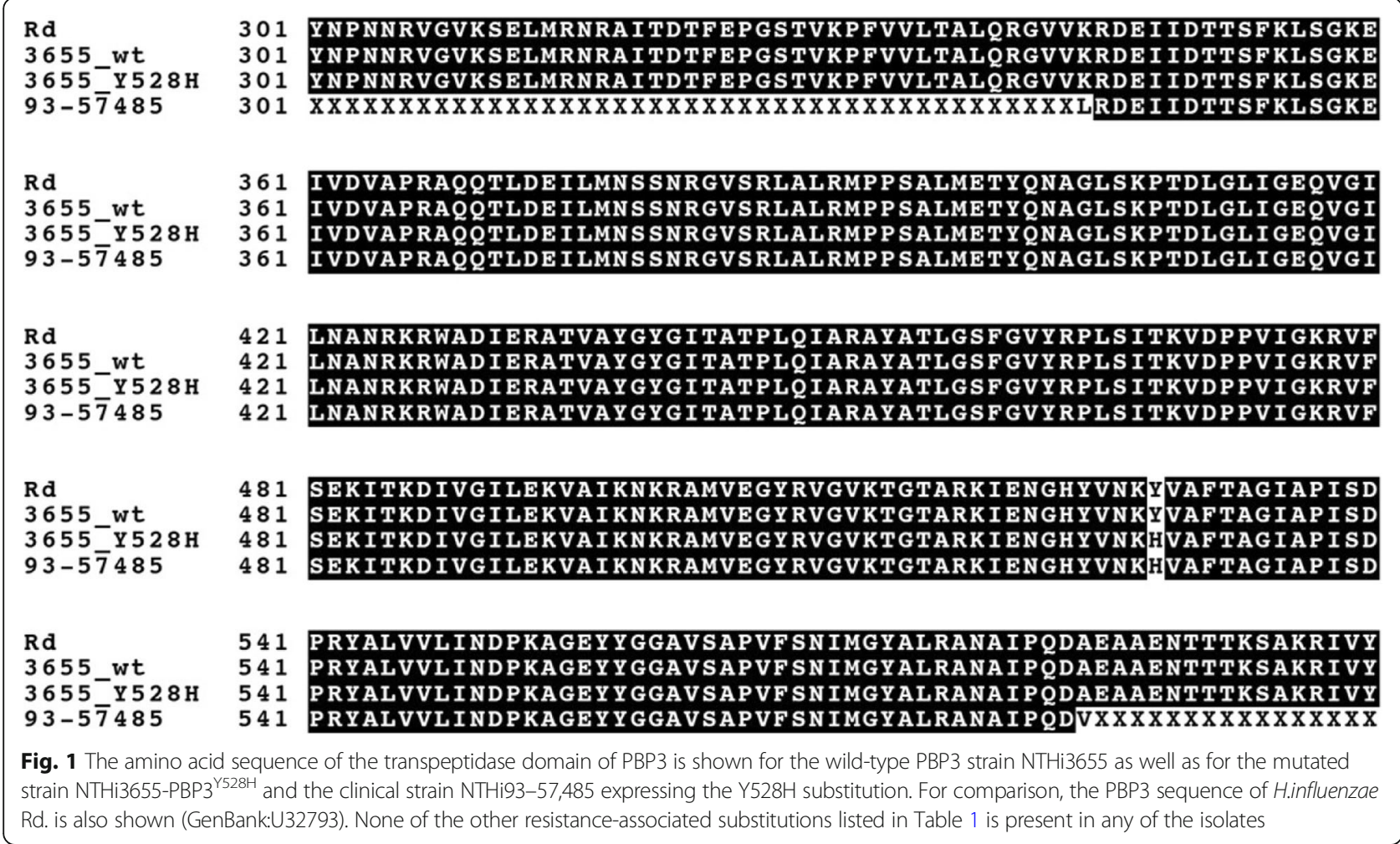

coli TOP10. The $f s t I$ gene was verified by DNA sequencing (Eurofins Genomics, Ebersberg, Germany). Site-directed mutagenesis was carried out using Pfu Turbo DNA polymerase (Agilent, Santa Clara, CA) and primers outlined in Table 2. The PCR products were digested using DpnI (Thermo Scientific, Waltham, MA) for $1 \mathrm{~h}$ at $37^{\circ} \mathrm{C}$. The mutated ftsI gene with confirmed mutation of $\mathrm{Y} 528 \mathrm{H}$ was amplified by PCR and transformed into the recipient strain NTHi3655 using the protocol by Poje and Redfield [13]. The generated mutant (named NTHi3655-PBP3 ${ }^{\mathrm{Y} 28 \mathrm{H}}$ ) was selected on BHI agar containing NAD and hemin, and increasing concentrations of ampicillin (0, 0.125, 0.25, 0.5, 0.75, 1 and $2 \mathrm{mg} / \mathrm{L}$, respectively). Finally, the $f t s I$ gene sequence in the resulting mutant was verified by DNA sequencing.

Antimicrobial susceptibility testing

Antimicrobial susceptibility testing was performed at the EUCAST development laboratory (Växjö, Sweden). Screening for $\beta$-lactam resistance with disc diffusion using $1 \mathrm{U}$ of benzylpenicillin (PcG) on fastidious Mueller Hinton (MH-F) solid medium was performed with NTHi93-57,485, NTHi3655 and NTHi3655-PBP3 ${ }^{Y 528 H}$ [10]. MICs to common $\beta$-lactam agents were also determined by broth microdilution (BMD) according to the

Table 2 Primers used for PCR amplification, site directed mutagenesis and sequencing of the fts/ and acrR genes

\begin{tabular}{|c|c|c|}
\hline Use of primers & Forward primer sequence & Reverse primer sequence \\
\hline Ampification of full length fts/ gene & 5' - CCTGCGTGTTTGAAAGTTGAAAGAGATG - 3' & $5^{\prime}$ - AACAAAGTAAGGGCGAGGATATTCCCAAAG - 3' \\
\hline $\begin{array}{l}\text { Introduction of Y528H substitution }{ }^{\mathrm{a}} \text { in } \mathrm{fts} / \\
\text { in NTHi3655 }\end{array}$ & $\begin{array}{l}5^{\prime}-\text { GAAAATGGACATTATGTAAATAAGCATGTGGCAT } \\
\text { TTACTGCGGG - 3' }\end{array}$ & $\begin{array}{l}5^{\prime}-\text { CCCGCAGTAAATGCCACATGCTTATTTACATAAT } \\
\text { GTCCATTTC - 3' }\end{array}$ \\
\hline $\begin{array}{l}\text { Amplification and sequencing of the } a c r R \\
\text { gene }\end{array}$ & $5^{\prime}$ - TTGTGGGTTTACGGCTTACC - 3' & $5^{\prime}-$ CCGATGACACCGACAAAAAT - 3' \\
\hline $\begin{array}{l}\text { Sequencing of fts/ gene fw1 (using } \\
\text { primer walking) }\end{array}$ & $5^{\prime}$ - CCAATAAACTCTACAGTTAAATGCTCGC - 3' & \\
\hline $\begin{array}{l}\text { Sequencing of ftsl gene fw2 (using } \\
\text { primer walking) }\end{array}$ & $5^{\prime}$ - AGCGGACGATAAACACCGAAACTACCA - 3' & \\
\hline $\begin{array}{l}\text { Sequencing of ftsl gene fw3 (using } \\
\text { primer walking) }\end{array}$ & $5^{\prime}$ - ATACTTAAGGTAACATCTTGTGCATCATAT - 3' & \\
\hline
\end{tabular}

${ }^{a}$ Induces nucleotide substitution $1582 \mathrm{~T}>\mathrm{C}$ to change the codon from a tyrosine residue (TAT) to a histidine residue (CAT) 
ISO standard 20776-1 [14] using MH-F broth [15]. Absence of $\beta$-lactamase production was confirmed by a standard nitrocefin test [16].

\section{Growth curves and sequencing of the acrR gene}

A few colonies of NTHi were resuspended in supplemented BHI broth and diluted to a starting $\mathrm{OD}_{600}$ of 0.05 . The bacterial suspension was incubated at $37^{\circ} \mathrm{C}$ and $5 \% \mathrm{CO}_{2}$ at $200 \mathrm{rpm}$ and $\mathrm{OD}_{600}$ was measured at indicated time points. The acrR gene, which encodes a regulator of the AcrAB efflux pump was sequenced using primers stated in Table 2.

\section{Results}

Site directed mutagenesis of the susceptible strain NTHi3655 yielded a mutant (NTHi3655-PBP3 ${ }^{\mathrm{Y} 528 \mathrm{H}}$ ) with an identical transpeptidase PBP3 sequence to that of the clinical isolate NTHi93-57485 (Fig. 1). The introduction of the substitution $\mathrm{Y} 528 \mathrm{H}$ into a wtPBP3 rendered the mutant NTHi3655-PBP3 ${ }^{\mathrm{Y} 528 \mathrm{H}}$ positive in the disc diffusion $\beta$-lactam resistance screening algorithm (Table 3). The mutant also demonstrated a one- or two-fold increase in MICs for aminopenicillins as revealed by susceptibility testing with broth microdilution (Table 3). However, the clinical isolate NTHi93-57485 still had a higher MIC for ampicillin $(1 \mathrm{mg} / \mathrm{L})$ and cefuroxime $(4 \mathrm{mg} / \mathrm{L})$ compared with NTHi3655-PBP3 ${ }^{\mathrm{Y} 528 \mathrm{H}}$. All strains were $\beta$-lactamase negative. None of the isolates had any frame shift insertions in the acrR gene.

According to BMD, all isolates had MICs that were below current clinical breakpoints proposed by EUCAST, contrary to the initial Etest results obtained with NTHi93-57485. It has previously been suggested that gradient tests have a tendency to overestimate MICs in H. influenzae with modified PBP3 [17].

\section{Discussion}

The introduction of the amino acid substitution $\mathrm{Y} 528 \mathrm{H}$ rendered a fully susceptible isolate to become positive in the benzylpenicillin screening test. It did not, however, restore zone or MIC levels of ampicillin to those of the clinical isolate NTHi93-57485. These results mimic the findings from site-direction mutagenesis experiments performed on substitutions N526K and R517H by Osaki et al. [9]. Our results further support the observation that mechanisms other than rPBP3, $\beta$-lactamase production or dysregulation of the AcrAB efflux pump affect susceptibility to $\beta$-lactams in $H$. influenzae. Interestingly, we also noted a reduced growth rate in NTHi93-57485 (Additional file 1) compared with NTHi3655 and NTHi3655-PBP3 ${ }^{\mathrm{Y} 528 \mathrm{H}}$. Decreased fitness as shown by slower growth rates has been shown to correlate with antimicrobial resistance in other bacterial species [18].
When Osaki et al. introduced the key residue substitution of N526K into the PBP3 of $H$. influenzae Rd. strain, ampicillin MIC increased only 1-fold, in good agreement with our current findings in NTHi3655-PBP3 ${ }^{\mathrm{Y} 528 \mathrm{H}}$ [9]. Therefore, despite the fact that these two main mutations near the KTG-motif managed to reduce aminopenicillin susceptibility, it seems that additional factors (PBP3-related or unrelated) are required for resistance surpassing clinical breakpoints. However, the prevalence of the substitution $\mathrm{Y} 528 \mathrm{H}$ in clinical isolates seem to be distinctly lower compared with N526K. To the best of our knowledge, the $\mathrm{Y} 528 \mathrm{H}$ substitution has only been sporadically described in studies where PBP3 has been sequenced, for instance, in two cefuroxime-resistant isolates where it appeared together with N526K and S357N [19]. A BLAST-search on publicly available PBP3 sequences on NCBI only identified one other isolate with this mutation (accession no. BAZ92405.1). The Y528H substitution is not included in the PBP3 substitutions previously investigated by site directed mutagenesis [9]. The reasons why this mutation seems less prevalent remain to be elucidated. Its introduction into PBP3 did not affect the growth rate of our experimental strain, but other manifestations of reduced bacterial fitness caused by this mutation still have to be conclusively ruled out. Also, it cannot be ruled out that the studied mutation is less efficient and thus less prone to selection by antibiotic treatment. It can be added to this discussion that the N526K substitution is rarely seen as a lone substitution in PBP3 in clinical isolates with reduced susceptibility to aminopenicillins.

Even though additional factors may be needed for resistance according to clinical breakpoints, several prior studies have demonstrated the importance of alterations in PBP3 for the development of $\beta$-lactam resistance in Haemophilus influenzae [3, 4, 9]. Group II strains, with a low-level aminopenicillin resistance, dominate in most studies $[1,7,20]$. 3D modelling has previously suggested that the N526K substitution lines the active site pocket of PBP3, near the catalytic motif of KTG-514 [3]. Given its proximity to this motif, it is likely that the $\mathrm{Y} 528 \mathrm{H}$ substitution also interferes with the active site pocket.

The proportional increase in recent years in $\mathrm{rPBP} 3$ strains together with increasing rates of resistance to sulfamethoxazole limits the number of treatment options for common respiratory tract infections, especially in children [20]. Also, current breakpoints for aminopenicillins assume intravenous dosage, whereas in everyday treatment of less severe infections, oral amoxicillin is often used. Pharmacokinetic simulations suggest that even a high oral amoxicillin dose $(750 \mathrm{mg}$ tid) does not always result in $40 \% f \mathrm{~T}>\mathrm{MIC}$ (including 2 standard deviations) for an isolate with an MIC of $1 \mathrm{mg} / \mathrm{L}$ [21]. Due to this, there is debate whether low-rPBP3 strains that are considered as susceptible according to clinical 


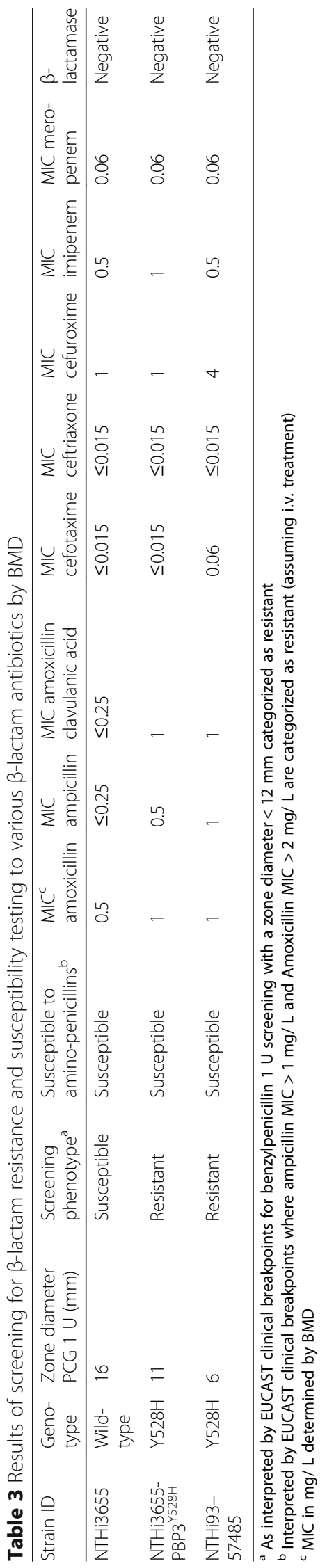


breakpoints can be safely treated with oral amoxicillin in clinical practice, where a dose of $500 \mathrm{mg}$ tid is commonly used. Until clinical studies have been performed to address this issue, there is a case for inclusive screening regimes.

\section{Conclusions}

In conclusion, we have identified a novel PBP3-mutation, $\mathrm{Y} 528 \mathrm{H}$, that affects aminopenicillin susceptibility in $H$. influenzae, and this mutation should be added to rPBP3-defining substitutions. It is clear that mechanisms other than $\beta$-lactamase production, point mutations in PBP3 or dysregulation of the AcrAB efflux pump can contribute to reduced $\beta$-lactam susceptibility.

\section{Additional file}

Additional file 1: Growth curves demonstrate that the clinical strain NTHi93-57,485 has a slower growth rate than the rPBP3 WT strain NTHi3655 and the corresponding mutant NTHi3655-PBP3 ${ }^{\mathrm{Y} 528 \mathrm{H}}$. Mean values from 3 separate experiments are shown. Error bars represent standard error of the mean (SEM). Circles represent NTHi3655, squares represent NTHi3655-PBP3 ${ }^{\mathrm{Y} 528 \mathrm{H}}$ and triangles represent NTHi93-57,485. (DOCX $72 \mathrm{~kb}$ )

\section{Abbreviations}

BHI: brain heart infusion (broth and solid medium); BMD: Broth Microdilution; EUCAST: European Committee on Antimicrobial Susceptibility Testing; $f T$ > MIC: The amount of time in which free or non-protein-bound antimicrobial concentration exceeds the minimum inhibitory concentration (MIC) of the organism; MALDI-TOF: Matrix Assisted Laser Desorption lonization - Time of Flight; MH-F: fastidious Mueller Hinton (broth and solid medium); MIC: Minimal inhibitory concentration; NAD: nicotinamide adenine dinucleotide; NTHi: non-typeable Haemophilus influenzae; PBP3: Penicillin Binding Protein 3; PCG: Benzylpenicillin; rPBP3: PBP3 mediated resistance present ('Resistant' PBP3); wtPBP3: wild type PBP3

\section{Acknowledgements}

The authors would like to thank Stina Bengtsson who did the initial sequencing of the transpeptidase domain of the $\mathrm{fts} / \mathrm{gene}$ in the isolate NTHi93-57485

\section{Funding}

This work was supported by grants from Foundation of Anna and Edwin Berger; the Swedish Medical Research Council (grant number K2015-57X03163-43-4, http://www.vr.se); Forssman's foundation (Physiographical Society Lund) and Skåne County Council's research and development foundation. The study bodies had no role in study design, sample collection, analysis of data or writing of the manuscript.

\section{Availability of data and materials}

The nucleotide sequences of $\mathrm{fts} /$ in the isolates NTHi3655-PBP3 ${ }^{\mathrm{Y} 528 \mathrm{H}}$ and NTHi93-57,485 have been deposited in GenBank under accession no. MG366893 and MG366894. The nucleotide sequences of acrR for isolates NTHi93-57,485 and NTHi3655-PBP3 ${ }^{\mathrm{Y} 528 \mathrm{H}}$ have been deposited in GenBank under accession no. MG366895 and MG366896.

\section{Authors' contributions}

$\mathrm{JT}$ conducted all the experiments apart from the antimicrobial susceptibility testing, analyzed the data and wrote the first draft of the manuscript, EM performed the antimicrobial susceptibility testing, YCS and KR contributed to study design and critically reviewed the manuscript, FR conceived and coordinated the study. All authors have read and approved the manuscript.
Ethics approval and consent to participate

Not applicable. Only microorganisms and no human material were handled as part of this project.

\section{Competing interests}

The authors declare that they have no competing interests.

\section{Publisher's Note}

Springer Nature remains neutral with regard to jurisdictional claims in published maps and institutional affiliations.

\section{Author details}

${ }^{1}$ Clinical Microbiology, Department of Translational Medicine, Lund University, Jan Waldenströms gata 59, SE-205 02 Malmö, Sweden. EUCAST Development Laboratory, Central Hospital Växjö, SE-351 85 Växjö, Sweden.

Received: 5 January 2018 Accepted: 23 May 2018

Published online: 31 May 2018

References

1. Tristram S, Jacobs MR, Appelbaum PC. Antimicrobial resistance in Haemophilus influenzae. Clin Microbiol Rev. 2007;20(2):368-89.

2. Kaczmarek FS, Gootz TD, Dib-Hajj F, Shang W, Hallowell S, Cronan M. Genetic and molecular characterization of beta-lactamase-negative ampicillin-resistant Haemophilus influenzae with unusually high resistance to ampicillin. Antimicrob Agents Chemother. 2004:48(5):1630-9.

3. Ubukata K, Shibasaki Y, Yamamoto K, Chiba N, Hasegawa K, Takeuchi Y, Sunakawa K, Inoue M, Konno M. Association of amino acid substitutions in penicillin-binding protein 3 with beta-lactam resistance in beta-lactamasenegative ampicillin-resistant Haemophilus influenzae. Antimicrob Agents Chemother. 2001:45(6):1693-9.

4. Hotomi M, Fujihara K, Billal DS, Suzuki K, Nishimura T, Baba S, Yamanaka N. Genetic characteristics and clonal dissemination of beta-lactamase-negative ampicillin-resistant Haemophilus influenzae strains isolated from the upper respiratory tract of patients in Japan. Antimicrob Agents Chemother. 2007; 51(11):3969-76.

5. Garcia-Cobos S, Campos J, Lazaro E, Roman F, Cercenado E, Garcia-Rey C, Perez-Vazquez M, Oteo J, de Abajo F. Ampicillin-resistant non-betalactamase-producing Haemophilus influenzae in Spain: recent emergence of clonal isolates with increased resistance to cefotaxime and cefixime. Antimicrob Agents Chemother. 2007;51(7):2564-73.

6. Dabernat H, Delmas C, Seguy M, Pelissier R, Faucon G, Bennamani S, Pasquier C. Diversity of beta-lactam resistance-conferring amino acid substitutions in penicillin-binding protein 3 of Haemophilus influenzae. Antimicrob Agents Chemother. 2002;46(7):2208-18.

7. Skaare D, Allum AG, Anthonisen IL, Jenkins A, Lia A, Strand L, Tveten Y, Kristiansen BE. Mutant ftsl genes in the emergence of penicillin-binding protein-mediated beta-lactam resistance in Haemophilus influenzae in Norway. Clin Microbiol Infec. 2010;16(8):1117-24.

8. Skaare D, Lia A, Hannisdal A, Tveten Y, Matuschek E, Kahlmeter G, Kristiansen BE. Haemophilus influenzae with non-Beta-lactamase-mediated Beta-lactam resistance: easy to find but hard to categorize. J Clin Microbiol. 2015:53(11):3589-95

9. Osaki Y, Sanbongi Y, Ishikawa M, Kataoka H, Suzuki T, Maeda K, Ida T. Genetic approach to study the relationship between penicillin-binding protein 3 mutations and Haemophilus influenzae beta-lactam resistance by using site-directed mutagenesis and gene recombinants. Antimicrob Agents Chemother. 2005;49(7):2834-9.

10. The European Committee on Antimicrobial Susceptibility Testing Breakpoint tables for interpretation of MICs and zone diameters. Version. 8: 0. http://www.eucast.org/

11. Sondergaard A, Petersen MT, Fuursted K, Norskov-Lauritsen N. Detection of N526K-substituted penicillin-binding protein 3 conferring low-level mutational resistance to beta-lactam antibiotics in Haemophilus influenzae by disc diffusion testing on Mueller-Hinton agar according to EUCAST guidelines. The J Antimicrob Chemother. 2012;67(6):1401-4.

12. Melhus A, Janson H, Westman E, Hermansson A, Forsgren A, Prellner K. Amoxicillin treatment of experimental acute otitis media caused by Haemophilus influenzae with non-beta-lactamase-mediated resistance to beta-lactams: aspects of virulence and treatment. Antimicrob Agents Chemother. 1997;41(9):1979-84. 
13. Poje G, Redfield RJ. Transformation of Haemophilus influenzae. Methods in molecular medicine. 2003;71:57-70.

14. International Standards Organisation. Reference method for testing the in vitro activity of antimicrobial agents against rapidly growing aerobic bacteria involved in infectious diseases. ISO. 1996:20776-1.

15. The European Committee on Antimicrobial Susceptibility Testing. Media preparation for EUCAST disk diffusion testing and for determination of MIC values by the broth microdilution method. v 4.0, 2014. http://www.eucast.org/, last Accessed 03 Mar 2017.

16. O'Callaghan CH, Morris A, Kirby SM, Shingler AH. Novel method for detection of beta-lactamases by using a chromogenic cephalosporin substrate. Antimicrob Agents Chemother. 1972; (4):283-8.

17. Karlsson C VA, Matuschek E, Skaare D and Kahlmeter G. : Evaluation of Etest, M.I.C.E. and MIC Test Strip beta-lactam gradient tests for beta-lactamase negative Haemophilus influenzae with beta-lactam resistance due to PBP3 substitutions. 26th ECCMID, Amsterdam, the Netherlands, 2016.

18. Andersson DI, Hughes D. Antibiotic resistance and its cost: is it possible to reverse resistance? Nat Rev Microbiol. 2010;8(4):260-71.

19. Straker K, Wootton M, Simm AM, Bennett PM, MacGowan AP, Walsh TR. Cefuroxime resistance in non-beta-lactamase Haemophilus influenzae is linked to mutations in ftsl. J Antimicrob Chemother. 2003;51(3):523-30.

20. Resman F, Ristovski M, Forsgren A, Kaijser B, Kronvall G, Medstrand P, Melander E, Odenholt I, Riesbeck K. Increase of beta-lactam-resistant invasive Haemophilus influenzae in Sweden, 1997 to 2010. Antimicrob Agents Chemother. 2012;56(8):4408-15.

21. de Velde F, de Winter BC, Koch BC, van Gelder T, Mouton JW. Non-linear absorption pharmacokinetics of amoxicillin: consequences for dosing regimens and clinical breakpoints. J Antimicrob Chemother. 2016;71(10): 2909-17.

Ready to submit your research? Choose BMC and benefit from:

- fast, convenient online submission

- thorough peer review by experienced researchers in your field

- rapid publication on acceptance

- support for research data, including large and complex data types

- gold Open Access which fosters wider collaboration and increased citations

- maximum visibility for your research: over $100 \mathrm{M}$ website views per year

At BMC, research is always in progress.

Learn more biomedcentral.com/submissions 